\title{
NORMAL MODES, UNCOUPLING, AND STABILITY FOR A CLASS OF NONLINEAR OSCILLATORS*
}

\author{
BY \\ G. PECELLI ${ }^{1,2}$ AND E. S. THOMAS $S^{1,3}$ \\ SUNY at Albany
}

1. Preliminaries. The concept of normal mode vibrations in mechanical systems originates with the idea of finding the fundamental frequencies of a linear system and introducing normal coordinates which decouple the system. We begin with a Hamiltonian $H(x, y): \mathbb{R}^{2 n} \rightarrow \mathbb{R}$, which, expanded in a Taylor series, has the form

$$
H(x, y)=H_{2}(x, y)+H_{3}(x, y)+\cdots,
$$

where $H_{2}(x, y)=\sum_{i=1}^{n}\left(\alpha_{i} / 2\right) y_{i}^{2}+V_{2}(x), \alpha_{i}>0$ for all $i$ and with $V_{2}(x)$ a positive definite quadratic form in the $n$ variables $x_{1}, \cdots, x_{n}$. It is well known that in this case we can find a linear canonical transformation which takes $H_{2}(x, y)$ to

$$
\tilde{H}_{2}(\tilde{x}, \tilde{y})=\sum_{i=1}^{n}\left(\lambda_{i} / 2\right)\left(\tilde{x}_{i}^{2}+\tilde{y}_{i}^{2}\right),
$$

where $\lambda_{i}>0$ for all $i$. If we assume for a moment that $H_{3}$ and all the higher-order terms vanish identically, we can define the normal modes via the invariant eigenspaces, on which the flow is circular. If we fix an energy surface $\tilde{H}_{2}(\tilde{x}, \tilde{y})=h$, we immediately obtain $n$ circular solutions, each of energy $h$. These are the normal modes, and their frequencies of vibration are the fundamental frequencies. If the higher-order terms do not vanish, the construction above can no longer be performed. In the case of low energy and positive definite $H_{2}(x, y)$, Weinstein [23] has proven the existence of periodic solutions continuing the modes. In the case of arbitrary energy the only results available seem to be the ones of Seifert [21], Rosenberg [14, 17] and Gordon [7], but the solutions so obtained may or may not be "modes" in any recognizable sense. Following Rosenberg [10, 12], we shall adopt a definition of "normal mode" as being that of a solution $\left(x_{1}(t), x_{2}(t), \cdots, x_{n}(t), y_{1}(t), \cdots\right.$, $\left.y_{n}(t)\right)$ of

$$
\dot{x}=H_{y}, \quad \dot{y}=-H_{x} \quad\left(H_{x}(0,0)=H_{y}(0,0)=0\right)
$$

satisfying:

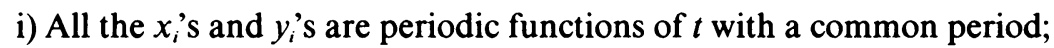

ii) There exists a time $t_{0}$ such that $x_{i}\left(t_{0}\right)=0$ for all $i$;

iii) There exists a time $t_{1} \neq t_{0}$ such that $y_{i}\left(t_{1}\right)=0$ for all $i$.

* Received January 1, 1978; revised version received October 12, 1978.

' Both authors partially supported by NSF grant MCS 78-01504.

${ }^{2}$ On leave from Hunter College, CUNY.

${ }^{3}$ Partially supported by the Alexander von Humboldt Foundation at the University of Würzburg. 
This definition is too general since it allows, in the linear case, for periodic solutions which are not modes [16]. We thus add a further condition:

iv) The variational equation $\dot{Z}=J H_{* *}(x(t), y(t), y(t)) Z$ (where $H_{* *}$ is the Hessian of $H$ ) uncouples, via a canonical transformation, i.e. there exists a canonical transformation $(\xi, \eta)=S(x, y)$ taking $H(x(t), y(t))$ to $\hat{H}(\xi(t), \eta(t))$, with $\xi$ and $\eta$ satisfying i)-iii) in place of $x$ and $y$ respectively and such that $\dot{Z}=J \hat{H}_{* *}(\xi(t), \eta(t)) Z$ with $J \hat{H}_{* *}(\xi(t), \eta(t))$ of the form

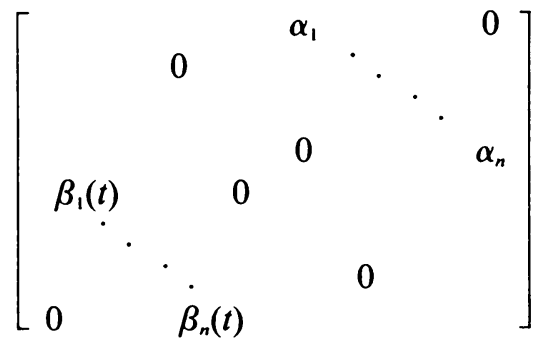

where $\alpha_{1}, \cdots, \alpha_{n}$ are constants and $\beta_{1}(t), \cdots, \beta_{n}(t)$ are periodic functions with the same period.

Although this last condition does not necessarily eliminate all solutions which are not modes, it provides a computationally useful condition automatically satisfied by the classical modes of linear systems. We shall not investigate existence of modes in the most general setting, although it would be of considerable interest to know whether the modes shown to exist by Rosenberg [14, 17, 19] and Cooke and Struble [6] also satisfy our condition (iv). We shall instead apply this definition to the study of isoenergetic stability of some special modes of vibration for a class of simple conservative nonlinear oscillators.

To be more specific, we shall study the isoenergetic stability of the two-degree-of-freedom oscillator with Hamiltonian

$$
\begin{aligned}
H(x, y) & =\left(\alpha_{1} / 2\right) y_{1}{ }^{2}+\left(\alpha_{2} / 2\right) y_{2}{ }^{2}+(1 / 2)\left(a_{1} x_{1}{ }^{2}+a_{2} x_{2}{ }^{2}\right) \\
& +\left(a_{3} / 2\right)\left(x_{1}-x_{2}\right)^{2}+(1 / 4)\left(b_{1} x_{1}{ }^{4}+b_{2} x_{2}{ }^{4}+b_{3}\left(x_{1}-x_{2}\right)^{4}\right) .
\end{aligned}
$$

We can think of this oscillator as being realized by the frictionless spring system of Fig. 1, where $x_{1}$ and $x_{2}$ are the displacements from rest of the masses $\alpha_{1}$ and $\alpha_{2}, \dot{x}_{i}=\alpha_{i} y_{i}, i=1,2$, and the restoring forces are

$$
F_{i}(\Delta)=a_{i} \Delta+b_{i} \Delta^{3}, \quad i=1,2,3 ;
$$

$a_{i}>0, b_{i}>0, i=1,2 ; a_{3} \geq 0, b_{3} \geq 0$ (not both zero).

In case the outer springs are identical and $\alpha_{1}=\alpha_{2}=1$, we have two natural periodic solutions, the in-phase oscillation $\left(x_{1} \equiv x_{2}\right)$, and the out-of-phase one $\left(x_{1} \equiv-x_{2}\right)$. It was incidentally shown in [11] that these two oscillations are indeed modes in the sense of (i)(iv), and their isoenergetic stability was studied in considerable detail. It should be clear that without such symmetry, the obvious candidates no longer exist, but fortunately they can be replaced by some fairly simple motions.

Definition 1.1. For the energy function

$$
H(x, y)=\left(\alpha_{1} / 2\right) y_{1}^{2}+\left(\alpha_{2} / 2\right) y_{2}^{2}+V\left(x_{1}, x_{2}\right),
$$




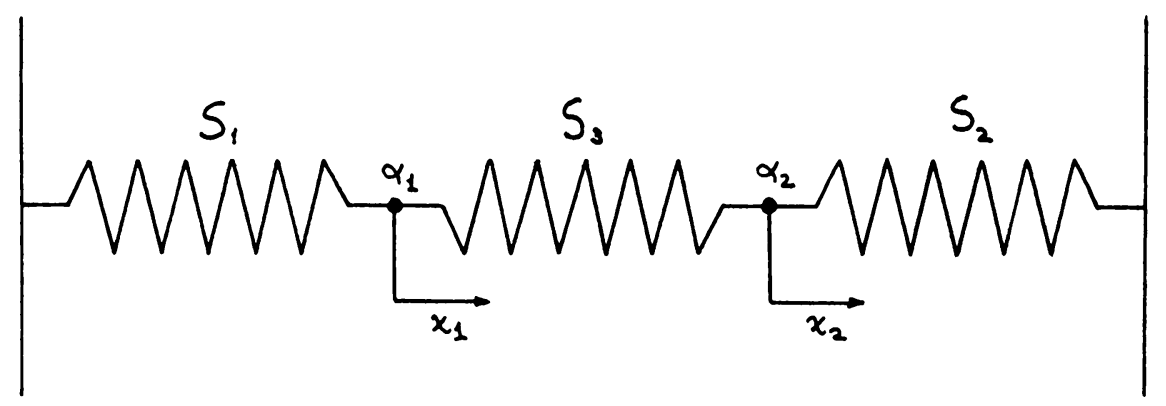

FIG. 1.

where $V(x)$ is sufficiently differentiable, we say that a nonstationary periodic solution $x(t)$ of

$$
\ddot{x}_{1}=-\alpha_{1} V_{x_{1}}, \quad \ddot{x}_{2}=-\alpha_{2} V_{x_{2}},
$$

is an (similar) in-phase mode for the Hamiltonian system

$$
\begin{gathered}
\left(\begin{array}{c}
\dot{x} \\
\dot{y}
\end{array}\right)=J H_{*}(x, y), \\
J=\left(\begin{array}{cc}
0 & I_{2} \\
-I_{2} & 0
\end{array}\right),
\end{gathered}
$$

$H_{*}=\operatorname{grad} H$, if $(x(t), \dot{x}(t))$ satisfies conditions (i)-(iii) above; if there exists $k>0$ such that $x_{1}(t) \equiv k x_{2}(t)$ and if (iv) above is satisfied with a linear canonical transformation. We have an (similar) out-of-phase mode when $k<0$. We further say that two modes coexist if the same linear canonical transformation uncouples the variational equations of both modes.

As we shall see in the development, certain restrictions on the oscillator are required for the coexistence of (similar) in- and out-of-phase modes. Furthermore, no analysis will be attempted for modes that are obtained as perturbation of similar modes by small changes of parameters [19]. Nevertheless, a surprising richness of behavior is still possible, even in the relatively simple case $\alpha_{1}=\alpha_{2}=1$, for which we will carry out a detailed analysis of the in-phase mode.

Some of our conclusions on the stability of the periodic orbits under study will be based on the following Arnold-Moser-Rüssmann criterion [1, 13, 20]. Let $H$ consist of those complex numbers $\mu$ of modulus one such that for some positive constants $c, \nu$ we have

$$
\left|\mu^{n}-1\right|>c n^{-v} \text { for } n=1,2,3, \cdots
$$

The complement of $H$ in the unit circle has measure 0 . Consider an area-preserving real analytic map $P: R^{2} \rightarrow R^{2}$ of the form

$$
P:\left\{\begin{array}{c}
x_{1}=a x+b y+\cdots \\
y_{1}=c x+d y+\cdots
\end{array}\right.
$$

where the dots represent power series beginning with terms of order at least two and converging in some neighborhood of the origin. Then we have: 
THEOREM 1.1. If the eigenvalues of the linear part of $P$ are complex conjugate (thus of modulus one) and belong to $H$, then the origin is a stable fixed point of $P$.

Stability off a set of measure zero can be improved to stability off a closed set of measure zero in some cases. In the fifth section we show how to do this using the twist theorem of Kolmogorov-Arnold-Moser. The procedure involves computing a twist coefficient and analyzing the set where it vanishes. The main result here is Theorem 5.1. Roughly speaking, the result is that when there is a chance for stability (i.e., when the Poincaré map is not hyperbolic), then one has stability except for those parameters lying in a closed set of measure 0 . Some remarks concerning generalizations of these ideas are included. Most computations are relegated to the Appendix.

The second author wishes to acknowledge the support of the Alexander von Humboldt Foundation and the University of Würzburg during the period in which this research was initiated.

2. The Poincaré map: uncoupling, reduction of the number of equations and the eigenvalues of the linear part. We consider a Hamiltonian system with energy function

$$
H(x, y)=\frac{\alpha_{1}}{2} y_{1}^{2}+\frac{\alpha_{2}}{2} y_{2}^{2}+V(x),
$$

where $\alpha_{1}, \alpha_{2} \neq 0$, and $V(x)$ is sufficiently differentiable. Let $w(t)$ be a periodic solution of

$$
\left(\begin{array}{c}
\dot{x} \\
\dot{y}
\end{array}\right)=J H \cdot(x, y)
$$

with period $2 T>0$. The variational equation along this solution is

$$
\dot{Z}=J H_{* *}(w(t)) Z=\left[\begin{array}{llll}
0 & 0 & \alpha_{1} & 0 \\
0 & 0 & 0 & \alpha_{2} \\
-V_{* *}(w(t)) & 0 & 0 \\
& & 0 & 0
\end{array}\right] Z,
$$

where $Z=\left[z_{i j}\right] ; i, j=1,2,3,4 ; Z(0)=I_{4}$ and $H_{* *}$ and $V_{* *}$ are the Hessians of $H$ and $V$ respectively. The system (2.2) is equivalent to the system of second-order scalar differential equations

$$
\ddot{z}_{1}=-\alpha_{1}\left[V_{11} z_{1}+V_{12} z_{2}\right], \quad \ddot{z}_{2}=-\alpha_{2}\left[V_{12} z_{1}+V_{22} z_{2}\right],
$$

with appropriate initial conditions and $V_{i j}=\left(\partial^{2} V / \partial x_{i} \partial x_{j}\right)(w(t))$.

THEOREM 2.1. There exists a real linear change of variables with constant coefficients

$$
U_{1}=a z_{1}+b z_{2}, \quad U_{2}=c z_{1}+d z_{2}
$$

with $a d-b c=1$, which transforms (2.3) into a system of the form

$$
U_{1}+P(t) U_{1}=0, \quad \ddot{U}_{2}+Q(t) U_{2}=0
$$

(with $P$ and $Q$ periodic of period $2 T$ ) if and only if

i) $V_{12} \equiv 0$ on $w(t)$

or 
ii) There exists a constant $K$ such that $\alpha_{1} V_{11}-\alpha_{2} V_{22} \equiv K V_{12}$ along $w(t)$, and $K^{2}+$ $4 \alpha_{1} \alpha_{2}>0$.

Remarks. (1) The theorem holds, modulo the periodicity of $P$ and $Q$, for any solution $w(t)$, periodic or not. It will generally hold along a solution that lies on a gradient line of the potential, although this by no means exhausts its applicability.

(2) The condition $K^{2}+4 \alpha_{1} \alpha_{2}>0$ is clearly redundant if $\alpha_{1}$ and $\alpha_{2}$ represent positive masses.

(3) In case (i) the identity transformation works.

(4) One of the referees suggested that this result is reminiscent of results of Hsu [9, 10], who obtained some conditions for a Hill's equation to be uncouplable via a transformation with constant coefficients. We observe that (2.3) under hypothesis (ii) can be rewritten as

$$
\left[\begin{array}{l}
\ddot{z}_{1} \\
\ddot{z}_{2}
\end{array}\right]+\left\{\alpha_{2} V_{22}(w(t))\left[\begin{array}{ll}
1 & 0 \\
0 & 1
\end{array}\right]+V_{12}(w(t))\left[\begin{array}{cc}
K & \alpha_{1} \\
\alpha_{2} & 0
\end{array}\right]\right\}\left[\begin{array}{l}
z_{1} \\
z_{2}
\end{array}\right]=0
$$

and can be seen to satisfy the conditions of Hsu [20], which are that in a vector Hill's equation of the form

$$
\ddot{z}+\left(\sum_{i=1}^{m} f_{i}(t) B_{i}\right) z=0
$$

one of the $B_{i}$ 's has distinct eigenvalues and all the $B_{i}$ 's commute. What our result shows is that, in case $\sum_{i=1}^{m} f_{i}(t) B_{i}$ arises from a potential function in two variables, then $m$ need be no larger than 2 with the $B_{i}$ 's having the form in $\left(2.3^{\prime}\right)$. Furthermore, our conditions are necessary and sufficient (while in Hsu [9] the conditions are not quite so) and are stated in terms of the potential function rather than the Hill's equation.

Proof. The "if" part of the statement (i) is obvious. To prove statement ii), differentiate (2.4) twice and use (2.3), obtaining

$$
\begin{aligned}
& \ddot{U}_{1}=-\left[\alpha_{1} a V_{11}+\alpha_{2} b V_{12}\right] z_{1}-\left[\alpha_{1} a V_{12}+\alpha_{2} b V_{22}\right] z_{2}, \\
& \ddot{U}_{2}=-\left[\alpha_{1} c V_{11}+\alpha_{2} d V_{12}\right] z_{1}-\left[\alpha_{1} c V_{12}+\alpha_{2} d V_{22}\right] z_{2} .
\end{aligned}
$$

If the transformation exists we must then have (since $z_{1}$ and $z_{2}$ are arbitrary)

$$
\begin{array}{ll}
\alpha_{1} a V_{11}+\alpha_{2} b V_{12}=a P(t) ; & \alpha_{1} a V_{12}+\alpha_{2} b V_{22}=b P(t), \\
\alpha_{1} c V_{11}+\alpha_{2} d V_{12}=c Q(t) ; & \alpha_{1} c V_{12}+\alpha_{2} d V_{22}=d Q(t) .
\end{array}
$$

If one of the constants $a, b, c$ or $d$ vanishes, then case (i) must hold. Thus we can assume $a b c d \neq 0$. We immediately obtain

$$
\begin{aligned}
& \left(\alpha_{1} a V_{11}+\alpha_{2} b V_{12}\right) / a=\left(\alpha_{1} a V_{12}+\alpha_{2} b V_{22}\right) / b \\
& \left(\alpha_{1} c V_{11}+\alpha_{2} d V_{12}\right) / c=\left(\alpha_{1} c V_{12}+\alpha_{2} d V_{22}\right) / d
\end{aligned}
$$

Some elementary algebra gives

$$
\begin{aligned}
\alpha_{1} V_{11}(w(t))-\alpha_{2} V_{22}(w(t)) & =\left[\left(\alpha_{1} a^{2}-\alpha_{2} b^{2}\right) / a b\right] V_{12}(w(t)) \\
& =\left[\left(\alpha_{1} c^{2}-\alpha_{2} d^{2}\right) / c d\right] V_{12}(w(t)) .
\end{aligned}
$$

Thus $K=\left(\alpha_{1} a^{2}-\alpha_{2} b^{2}\right) / a b=\left(\alpha_{1} c^{2}-\alpha_{2} d^{2}\right) / c d$. Some further algebra shows that $\alpha_{1} a c+$ 
$\alpha_{2} b d=0$ and $K^{2}+4 \alpha_{1} \alpha_{2}>0$. The "only if" part of the theorem is thus proven. To finish the proof of the "if" part, we must show that, given $K$, the constants $a, b, c, d$ can be chosen in an appropriate manner. We assume $K^{2}+4 \alpha_{1} \alpha_{2}>0$ and find $a, b, c, d$ so that $a d-$ $b c=1 ;\left(\alpha_{1} a^{2}-\alpha_{2} b^{2}\right) / a b=K=\left(\alpha_{1} c^{2}-\alpha_{2} d^{2}\right) / c d$ and $\alpha_{1} a c+\alpha_{2} b d=0$. With $b=\tau_{1} a$ we have $\tau_{1}=\left(-K \pm\left(K^{2}+4 \alpha_{1} \alpha_{2}\right)^{1 / 2}\right) / 2 \alpha_{2}$, and with $d=\tau_{2} c$ we have $\tau_{2}=\left(-K \pm\left(K^{2}+4 \alpha_{1} \alpha_{2}\right)^{1 / 2}\right.$ $/ 2 \alpha_{2}$. The other two algebraic relations require that $a c\left(\tau_{2}-\tau_{1}\right)=1$ and $a c\left(\alpha_{1}+\alpha_{2} \tau_{1} \tau_{2}\right)=0$. The second is satisfied by the choices

$$
\tau_{1}=\left(-K-\left(K^{2}+4 \alpha_{1} \alpha_{2}\right)^{1 / 2}\right) / 2 \alpha_{2}, \quad \tau_{2}=\left(-K+\left(K^{2}+4 \alpha_{1} \alpha_{2}\right)^{1 / 2}\right) / 2 \alpha_{2},
$$

or

$$
\tau_{1}=\left(-K+\left(K^{2}+4 \alpha_{1} \alpha_{2}\right)^{1 / 2}\right) / 2 \alpha_{2}, \quad \tau_{2}=\left(-K-\left(K^{2}+4 \alpha_{1} \alpha_{2}\right)^{1 / 2}\right) 2 \alpha_{2} .
$$

Since $\tau_{2}-\tau_{1}= \pm\left(\left(K^{2}+4 \alpha_{1} \alpha_{2}\right)^{1 / 2}\right) / \alpha_{2} \neq 0$, the constants $a, b, c, d$ can now be chosen (not necessarily in a unique manner). This completes the proof.

We observe that $P(t)=\alpha_{1} V_{11}+\tau_{1} \alpha_{2} V_{12}$ and $Q(t)=\alpha_{1} V_{11}+\tau_{2} \alpha_{2} V_{22}$; thus $P$ and $Q$ are determined up to interchange by choosing a sign for $\tau_{1}$. Some easy computations, using the expression for $K$ established in the proof and using condition (ii), give the following corollaries:

COROLlaRY 2.2. With $a, b, c, d$ as in Theorem 2.1, the transformation

$$
\xi_{1}=a x_{1}+b x_{2}, \quad \xi_{2}=c x_{1}+d x_{2} \quad \eta_{1}=d y_{1}-c y_{2}, \quad \eta_{2}=-b y_{1}+a y_{2},
$$

is canonical and takes the Hamiltonian (2.1) to

$$
\tilde{H}(\xi, \eta)=\frac{\tilde{\alpha}_{1}}{2} \eta_{1}{ }^{2}+\frac{\tilde{\alpha}_{2}}{2} \eta_{2}{ }^{2}+\tilde{V}(\xi)
$$

where $\tilde{\alpha}_{1}=\alpha_{1} a^{2}+\alpha_{2} b^{2}, \tilde{\alpha}_{2}=\alpha_{1} c^{2}+\alpha_{2} d^{2}$ and $\tilde{V}_{12} \equiv 0$ on the image of $w(t)$.

COROLLARY 2.3. Under the hypotheses of Corollary 2.2, the further canonical transformation

$$
\Theta_{1}=\left(\left|\tilde{\alpha}_{1}\right|\right)^{1 / 2} \xi_{1}, \quad \Theta_{2}=\left(\left|\tilde{\alpha}_{2}\right|\right)^{1 / 2} \xi_{2}, \quad \zeta_{1}=\eta_{1} /\left(\left|\tilde{\alpha}_{1}\right|\right)^{1 / 2}, \quad \zeta_{2}=\eta_{2} /\left(\left|\tilde{\alpha}_{2}\right|\right)^{1 / 2},
$$

takes (2.7) to the form

$$
\tilde{H}(\theta, \zeta)= \pm \frac{1}{2} \xi_{1}^{2} \pm \frac{1}{2} \zeta_{2}^{2}+\tilde{\tilde{H}}(\theta)
$$

where $\tilde{V}_{12} \equiv 0$ on $\omega(t)$, the final image of $w(t)$. Furthermore, the original variational equations reduce to the uncoupled second order linear system

$$
\ddot{U}_{1} \pm \tilde{\tilde{V}}_{11}(\omega(t)) U_{1}=0, \quad \ddot{U}_{2} \pm \tilde{\tilde{V}}_{22}(\omega(t)) U_{2}=0 .
$$

Since $Z(2 T)$ is the linearized Poincaré map [8, p. 253], it should be clear that, under the conditions of Theorem 2.1, a detailed study of its eigenvalues becomes substantially more feasible. It is well known that, if $w(t)$ lies on an energy surface $H(x, y)=h$, then $Z(2 T)$ has two eigenvalues equal to one. We now show what effect this has on the uncoupled equations of the linearized Poincare map. We state and prove a result for two degrees of freedom, the partial generalization to $n$ degrees of freedom being then obvious, a little messier and not as useful. We begin by stating two lemmas. 
LEMMA 2.4. Let $Z$ be a $4 \times 4$ matrix such that $\operatorname{det}(Z)=1$, with $\lambda=1$ as a double eigenvalue. Then the remaining two eigenvalues are given by

$$
\lambda=\frac{1}{2}\left[(D-2) \pm\left(D(D-4)^{1 / 2}\right)\right],
$$

where $D=$ Trace $(Z)$.

Proof. Straightforward elementary algebra.

LEMMA 2.5. Let $A(t)$ be periodic and even with period $p>0$. Let $u_{1}$ and $u_{2}$ be normalized solutions of $\ddot{u}+A(t) u=0$ (i.e. $\left.u_{1}(0)=\dot{u}_{2}(0)=1, \dot{u}_{1}(0)=u_{2}(0)=0\right)$. Then

i) $u_{1}(t \pm p)=u_{1}(p) u_{1}(t) \pm \dot{u}_{1}(p) u_{2}(t)$

ii) $u_{2}(t \pm p)= \pm u_{2}(p) u_{1}(t)+\dot{u}_{2}(p) u_{2}(t)$

iii) $u_{1}(t) \dot{u}_{2}(t)-\dot{u}_{1}(t) u_{2}(t) \equiv 1$

iv) $u_{1}(p)=\dot{u}_{2}(p)$.

Proof. Well-known and easy (see e.g. [2]).

THEOREM 2.6. If the conditions of Theorem 2.1 hold and $\tilde{V}_{11}(\omega(t)), \tilde{V}_{22}(\omega(t))$ are even functions of $t$, then Trace $Z(2 T)=2\left[U_{1}(2 T)+U_{2}(2 T)\right]$, where $U_{1}$ and $U_{2}$ are the solutions of (2.8) with $U_{1}(0)=U_{2}(0)=1, \dot{U}_{1}(0)=\dot{U}_{2}(0)=0$. Furthermore, if the period of both $\tilde{V}_{11}(\omega(t))$ and $\tilde{V}_{22}(\omega(t))$ is $T$, we have Trace $Z(2 T)=4\left[U_{1}^{2}(T)+U_{2}^{2}(T)-1\right]$.

Proof. Let

$$
M=\left[\begin{array}{cccc}
d /(|\tilde{\alpha}|)^{1 / 2} & -c /(|\tilde{\alpha}|)^{1 / 2} & 0 & 0 \\
-b /(|\tilde{\alpha}|)^{1 / 2} & a /(|\tilde{\alpha}|)^{1 / 2} & 0 & 0 \\
0 & 0 & a(|\tilde{\alpha}|)^{1 / 2} & b(|\tilde{\alpha}|)^{1 / 2} \\
0 & 0 & c(|\tilde{\alpha}|)^{1 / 2} & d(|\tilde{\alpha}|)^{1 / 2}
\end{array}\right]
$$

Let $U(t)=M Z(t)$. Then $U(t)$ satisfies the matrix equation

$$
\dot{U}=\left[\begin{array}{cccc}
0 & 0 & \pm 1 & 0 \\
0 & 0 & 0 & \pm 1 \\
-\tilde{V}_{11}(\omega(t)) & 0 & 0 & 0 \\
0 & -\tilde{V}_{22}(\omega(t)) & 0 & 0
\end{array}\right] U .
$$

Let $W(t)$ be the principal matrix solution of (2.9); then

$$
W(t)=\left[\begin{array}{cccc}
u_{1}(t) & 0 & u_{2}(t) & 0 \\
0 & v_{1}(t) & 0 & v_{2}(t) \\
\dot{u}_{1}(t) & 0 & \dot{u}_{2}(t) & \\
0 & \dot{v}_{1}(t) & 0 & \dot{v}_{2}(t)
\end{array}\right],
$$

where $u_{1}$ and $u_{2}$ are the normalized solutions of $\ddot{u}+\tilde{V}_{11}(\omega(t)) u=0$ and $v_{1}$ and $v_{2}$ are the normalized solutions of $\ddot{v}+\tilde{\tilde{V}}_{22}(\omega(t)) v=0$. ("Normalized" means that $u_{1}(0)=1=\dot{u}_{2}(0)$ and $\dot{u}_{1}(0)=0=u_{2}(0)$.) Since $U(t)$ satisfies (2.9), it is a constant multiple of $W(t)$ and, by initial data, we find that $W(t)=M Z(t) M^{-1}$; hence Trace $W(2 T)=$ Trace $Z(2 T)$. Using Lemma 2.5(iv) we obtain the first part of the theorem; using the full lemma with a bit of algebra we obtain the rest.

We are now ready to assume that the Poincaré map along $w(t)$ is restricted to an en- 
ergy surface $H(x, y)=h$. We also assume, for simplicity, that $\alpha_{1}$ and $\alpha_{2}$ are both positive. We begin with a Hamiltonian

$$
\tilde{\tilde{H}}(\theta, \zeta)=\frac{1}{2} \zeta_{1}^{2}+\frac{1}{2} \zeta_{2}^{2}+\tilde{\tilde{V}}\left(\Theta_{1}, \theta_{2}\right), \quad \tilde{\tilde{H}}(\Theta, \zeta)=h,
$$

which possesses a periodic orbit $\omega(t)$ of period $2 T$ and such that $\tilde{V}_{12} \equiv 0$ on $\omega(t)$. $\omega(0)$ is an arbitrary point on the orbit, $\omega(0)=\left(\theta_{1}(0), \theta_{2}(0), \zeta_{1}(0), \zeta_{2}(0)\right)$. By the third remark following Theorem 2.1, the matrices $Z(t)$ and $W(t)$ given by $(2.11)$ agree. It is well known that

$$
\mathrm{W}(2 T) \dot{\omega}(0)=\dot{\omega}(0), \quad H_{*}(\omega(0))_{*} W(2 T)=H_{*}(\omega(0))_{*}
$$

where $H_{*}(\omega(0))_{*}$ is the transpose of the gradient to the energy surface $H=h$ at the point $\omega(0)$. Writing (2.12) in scalar form and performing some elementary algebra, we obtain

$$
\begin{aligned}
{\left[u_{1}(2 T)+\dot{u}_{2}(2 T)-2\right] \zeta_{1}(0) } & =0, & & {\left[v_{1}(2 T)+\dot{v}_{2}(2 T)-2\right] \zeta_{2}(0)=0, } \\
{\left[u_{1}(2 T)+\dot{u}_{2}(2 T)-2\right] \tilde{V}_{1}\left(\Theta_{1}(0), \Theta_{2}(0)\right) } & =0, & & {\left[v_{1}(2 T)+\dot{v}_{2}(2 T)-2\right] \tilde{\tilde{V}}_{2}\left(\Theta_{1}(0), \Theta_{2}(0)\right)=0 . }
\end{aligned}
$$

Using Lemma 2.5 , we obtain

$$
\begin{aligned}
{\left[u_{1}(2 T)-1\right] \zeta_{1}(0)=0, } & {\left[v_{1}(2 T)-1\right] \zeta_{2}(0)=0, } \\
{\left[u_{1}(2 T)-1\right] \tilde{V}_{1}\left(\Theta_{1}(0), \Theta_{2}(0)\right)=0, } & {\left[v_{1}(2 T)-1\right] \tilde{\tilde{V}}_{2}\left(\Theta_{1}(0), \Theta_{2}(0)\right)=0 . }
\end{aligned}
$$

By recalling that $u_{1}(2 T)=U_{1}(2 T)$ and $v_{1}(2 T)=U_{2}(2 T)$, Theorem 2.6 and Lemma 2.4, plus the fact that $\omega(0)$ was an arbitrary point on the solution, we get:

COROLlARY 2.7. Suppose $H$ has form $(2.11)$ and $\tilde{\tilde{V}}_{12}$ vanishes identically on a periodic orbit $\omega(t)=\left(\Theta_{1}(t), \Theta_{2}(t), \zeta_{1}(t), \zeta_{2}(t)\right)$. Then this orbit is parabolic unless $\zeta_{1}(t) \equiv 0 \equiv \tilde{\tilde{V}}_{1}\left(\Theta_{1}(t)\right.$, $\left.\Theta_{2}(t)\right)$ or $\zeta_{2}(t) \equiv 0 \equiv \tilde{V}_{2}\left(\Theta_{1}(t), \Theta_{2}(t)\right)$.

Example. $H(x, y)=\frac{1}{2} y_{1}{ }^{2}+\frac{1}{2} y_{2}{ }^{2}-\cos x_{1}-\cos x_{2}$; the orbit in question satisfies $\ddot{x}_{1}+$ $\cos x_{1}=0, \ddot{x}_{2}+\cos x_{2}=0, x_{1} \equiv x_{2}$ (see Fig. 2). By Corollary 2.7 the parabolicity is immediate. That not all periodic orbits of Hamiltonians of the form

$$
\frac{1}{2} y_{1}^{2}+\frac{1}{2} y_{2}^{2}+f_{1}\left(x_{1}\right)+f_{2}\left(x_{2}\right)
$$

are parabolic can be seen by studying the orbit $\ddot{x}_{1}+\cos x_{1}=0, x_{2} \equiv 0$ of the example. This orbit will be elliptic or parabolic depending on the value of the total energy [4] (at least for those values of the energy which give rise to a periodic orbit).

COROLlaRY 2.8. In a Hamiltonian of the form

$$
\frac{1}{2} y_{1}^{2}+\frac{1}{2} y_{2}^{2}+f_{1}\left(x_{1}\right)+f\left(x_{2}\right)
$$

any nontrivial periodic orbit such that neither $x_{1}(t)$ nor $x_{2}(t)$ is constant is parabolic.

COROLLARY 2.9. If the periodic orbit $\omega(t)$ of $(2.11)$ is such that $\zeta_{2}(t) \equiv 0 \equiv \tilde{\tilde{V}}_{2}\left(\Theta_{1}(t), \theta_{2}(t)\right)$ and $\tilde{\tilde{V}}_{22}\left(\Theta_{1}(t), \Theta_{2}(t)\right)$ is an even function of $t$, the remaining two eigenvalues are given by

$$
\begin{gathered}
\lambda=U_{2}(2 T) \pm\left(U_{2}(2 T)^{2}-1\right)^{1 / 2} \text { if } \tilde{\tilde{V}}_{22}\left(\Theta_{1}(t), \Theta_{2}(t)\right) \text { has period } 2 T \\
\lambda=\left[U_{2}(T) \pm\left(U_{2}(T)^{2}-1\right)^{1 / 2}\right]^{2} \text { if } \tilde{\tilde{V}}_{22}\left(\Theta_{1}(t), \Theta_{2}(t)\right) \text { has period } T
\end{gathered}
$$




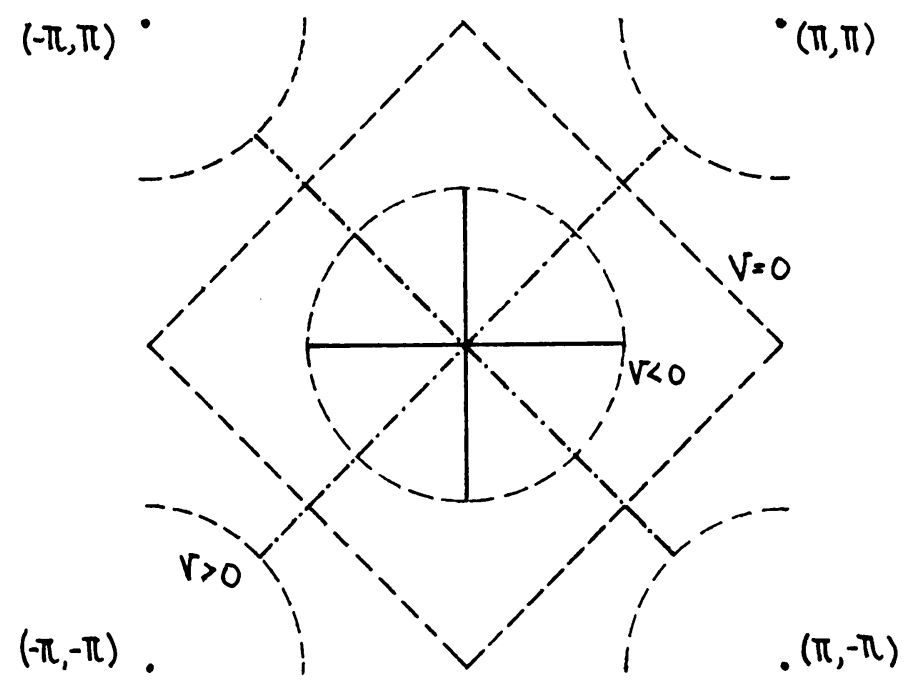

FIG. 2.

Proof. Lemma 2.4, Lemma 2.5, Theorem 2.6 and Corollary 2.7.

Remark. In general the solutions depend on some parameters, including the total energy of the system. If the dependence on parameters is analytic, we can thus conclude that, as the appropriate parameter changes, one of the Hill's equations (2.8) governing the linearized Poincare map will lie on a "boundary of instability", while the remaining equation will determine the character (elliptic, parabolic or hyperbolic) of the periodic orbit. Occasionally a further change of variables is needed to transform the final Hill's equation to a canonical form (Mathieu, Lamé, etc.) but it is clear that this will in no way alter its stability behavior.

3. Coexistence of modes and uncoupling of the variational equations for the oscillator (1.1). As an application of Theorem 2.1 we find relations among the constants of the Hamiltonian (1.1) which will guarantee the existence of "coexistent in- and out-of-phase modes." In case we do not ask for simultaneous uncoupling of the variational equations along the modes, many configurations are possible, with four, two or no modes at all. Whether orbits analogous to modes, over which the Poincare map can be uncoupled, will always appear, is an open question and any answer in this direction would be of interest.

In the next sections we will omit most of the details, with a warning to the reader that complete verification of all formulas, although elementary, is time-consuming.

THEOREM 3.1. In the oscillator with Hamiltonian (1.1) assume that $a_{3}>0$ and $a_{2} \geq a_{1}$. The oscillator will possess coexistent in- and out-of-phase modes along which the variational equations can be simultaneously uncoupled by a transformation with constant coefficients if and only if one of the following holds.

I. $b_{3}=0$; and

i) $\alpha_{1}=\alpha_{2}, a_{1}=a_{2}, b_{1}=b_{2}, k= \pm 1, a_{3}$ arbitrary; or

ii) $\alpha_{1} \neq \alpha_{2}, a_{2}>a_{1}, b_{2} / b_{1}=\alpha_{1}{ }^{2} / \alpha_{2}^{2}, k= \pm\left(\alpha_{1} / \alpha_{2}\right)^{1 / 2}, a_{3}=\left(\alpha_{2} a_{2}-\alpha_{1} a_{1}\right) /\left(\alpha_{1}-\alpha_{2}\right)>0$.

II. $b_{3}>0$; and

i) $\alpha_{1}=\alpha_{2}, a_{1}=a_{2}, b_{1}=b_{2}, k= \pm 1, a_{3}$ arbitrary or 
ii) $b_{2}-\eta b_{1}>0, b_{2}-\eta^{2} b_{1}>0, a_{2}-\eta a_{1}>0$,

$$
\begin{aligned}
& a_{3}=\left[\left(b_{2}+\eta b_{1}\right)\left(a_{2}-\eta a_{1}\right)\right] /\left[(1+\eta)\left(b_{2}-\eta b_{1}\right)\right], \\
& b_{3}=\left[\left(b_{2}+\eta b_{1}\right)\left(b_{2}-\eta^{2} b_{1}\right)\right] /\left[(1+\eta)^{2}\left(b_{2}-\eta b_{1}\right)\right], \text { and } \\
& k=\left[\left(b_{2}-\eta^{2} b_{1}\right) \pm\left((1+\eta)\left(b_{2}^{2}+\eta^{3} b_{1}^{2}\right)\right)^{1 / 2}\right] /\left(b_{2}+\eta b_{1}\right), \\
& \text { where } \eta=\alpha_{1} / \alpha_{2}, \text { or }
\end{aligned}
$$

iii) the configuration obtained by reversing inequalities in (ii).

Proof. We provide a sketch of the computations. The equations of motion are

$$
\begin{aligned}
& \ddot{x}_{1}=-\alpha_{1}\left[a_{1} x_{1}+a_{3}\left(x_{1}-x_{2}\right)+\left(b_{1} x_{1}{ }^{3}+b_{3}\left(x_{1}-x_{2}\right)^{3}\right)\right], \\
& \ddot{x}_{2}=-\alpha_{2}\left[a_{2} x_{2}-a_{3}\left(x_{1}-x_{2}\right)+\left(b_{2} x_{2}{ }^{3}-b_{3}\left(x_{1}-x_{3}\right)^{3}\right)\right] .
\end{aligned}
$$

Solutions of the form $x_{1}(t) \equiv k x_{2}(t)$ will exist if and only if the algebraic system (with $\eta=$ $\left.\alpha_{1} / \alpha_{2}\right)$

$$
\begin{gathered}
a_{3} k^{2}-\left[\left(a_{2}+a_{3}\right)-\eta\left(a_{1}+a_{3}\right)\right] k-\eta a_{3}=0, \\
b_{3} k^{4}+\left(\eta b_{1}+b_{3}(\eta-3)\right) k^{3}+3 b_{3}(1-\eta) k^{2}-\left(b_{2}+(1-3 \eta) b_{3}\right) k-\eta b_{3}=0
\end{gathered}
$$

is satisfied for some real $k$. Solving the first equation we have

$$
k=\left\{\left[\left(a_{2}+a_{3}\right)-\eta\left(a_{1}+a_{3}\right)\right] \pm\left(\left[\left(a_{2}+a_{3}\right)-\eta\left(a_{1}+a_{3}\right)\right]^{2}+4 \eta a_{3}^{2}\right)^{1 / 2}\right\} / 2 a_{3} .
$$

In applying Theorem 2.1 we observe that the condition $V_{12}\left(k x_{2}, x_{2}\right) \equiv 0$ can never be satisfied, since $a_{3}>0$, and we are led to the system

$$
\begin{gathered}
\left(a_{2}+a_{3}\right)-\eta\left(a_{1}+a_{3}\right)=K a_{3}, \\
\eta\left(b_{1} k^{2}+b_{3}(k-1)^{2}\right)-\left(b_{2}+b_{3}(k-1)^{2}\right)=-K b_{3}(k-1)^{2} .
\end{gathered}
$$

I. $b_{3}=0$. Both (3.2) and (3.4) imply that $\eta b_{1} k^{2}-b_{2}=0$. Hence $k_{1}, k_{2}= \pm\left(b_{2} / \eta b_{1}\right)^{1 / 2}$ in some order, so that $k_{1}+k_{2}=0$. Apply this to (3.3) to obtain $\left(a_{2}+a_{3}\right)-\eta\left(a_{1}+a_{3}\right)=0$. The claim follows, with $K=0$.

II. $b_{3}>0$. Eliminating $K$ in (3.4) and solving for $k$, we obtain, with $\sigma=a_{3} / b_{3}$,

$k=\left[\left(a_{2}-\eta a_{1}\right) \pm\left(\left(a_{2}-\eta a_{1}\right)^{2}+\left(\eta \sigma b_{1}-\eta a_{1}+a_{2}\right)\left(\sigma b_{2}-a_{2}\right)\right)^{1 / 2}\right] /\left(\eta \sigma b_{1}-\eta a_{1}+a_{2}\right)$,

where $\left(\eta \sigma b_{1}-\eta a_{1}+a_{2}\right)$ cannot be zero. Set the product of the roots (3.5) equal to $-\eta$ (by (3.3)) and write this as an equation in $\sigma$ to obtain

$$
\sigma\left(b_{2}-\eta^{2} b_{1}\right)+(\eta+1)\left(\eta a_{1}-a_{2}\right)=0 .
$$

If $b_{2}-\eta^{2} b_{1} \neq 0$, we have $\sigma=(\eta+1)\left(a_{2}-\eta a_{1}\right) /\left(b_{2}-\eta^{2} b_{1}\right)=a_{3} / b_{3}>0$; if $b_{2}-\eta^{2} b_{1}=0$, then $a_{1}=a_{2}$. Using the latter in (3.3) we obtain $k=1,-\eta$; using it in (3.5) we find that $k$ $= \pm\left(\eta \sigma^{2} b_{1} b_{2}\right)^{1 / 2} / \eta \sigma b_{1}$. The only way that $k_{1}$ and $k_{2}$ can have both forms is if $\eta=1$. Thus we have $\alpha_{1}=\alpha_{2}, a_{1}=a_{2}, b_{1}=b_{2}$, with $a_{3}$ arbitrary (the symmetric case [11]). Returning to the alternative $b_{2}-\eta^{2} b_{1} \neq 0$, we see that either $a_{2}-\eta a_{1}>0, b_{2}-\eta^{2} b_{1}>0$ or $a_{2}-\eta a_{1}<$ $0, b_{2}-\eta^{2} b_{1}<0$. Assume, for simplicity, the first alternative. Replace $\sigma$ in (3.5) to obtain

$$
k=\left[\left(b_{2}-\eta^{2} b_{1}\right) \pm\left((\eta+1)\left(b_{2}^{2}+\eta^{3} b_{1}^{2}\right)\right)^{1 / 2}\right] /\left(b_{2}+\eta b_{1}\right) .
$$

Using (3.3) and (3.6) we further compute

$$
\begin{gathered}
a_{3}=\left[\left(b_{2}+\eta b_{1}\right)\left(a_{2}-\eta a_{1}\right)\right] /\left[(1+\eta)\left(b_{2}-\eta b_{1}\right)\right], \\
b_{3}=\left[\left(b_{2}+\eta b_{1}\right)\left(b_{2}-\eta^{2} b_{1}\right)\right] /\left[(1+\eta)^{2}\left(b_{2}-\eta b_{1}\right)\right] .
\end{gathered}
$$


So the linear and nonlinear terms of the coupling spring are uniquely fixed by the remaining parameters. We also observe the further condition $b_{2}-\eta b_{1}>0\left(b_{2}-\eta b_{1}=0\right.$ would imply $a_{2}-\eta a_{1}=0$ and this would revert us to Case I). Notice that $b_{3}$ depends only on the mass ratio and the nonlinear terms. Replacing $b_{3}$ in (3.2) via (3.8) we compute

$$
\begin{gathered}
k^{4}+\frac{(\eta-1)\left(b_{2}+\eta b_{1}\right)^{2}-2\left(b_{2}-\eta^{2} b_{1}\right)^{2}}{\left(b_{2}+\eta b_{1}\right)\left(b_{2}-\eta^{2} b_{1}\right)} k^{3}+3(1-\eta) k^{2} \\
-\frac{\eta(\eta-1)\left(b_{2}+\eta b_{1}\right)^{2}+2\left(b_{2}-\eta^{2} b_{1}\right)^{2}}{\left(b_{2}+\eta b_{1}\right)\left(b_{2}-\eta^{2} b_{1}\right)} k-\eta=0,
\end{gathered}
$$

which can be shown by long division to have the two real solutions (3.6), thus adding no further constraints.

4. Lamé's equation and a stability theorem. We now undertake a detailed study of the in-phase-mode in the case $\alpha_{1}=\alpha_{2}=1, a_{2}>a_{1}, b_{2}>b_{1}>0, a_{3}=\left[\left(a_{2}-a_{1}\right)\left(b_{2}+b_{1}\right)\right] /$ $2\left(b_{2}-b_{1}\right)>0, b_{3}=\left(b_{2}+b_{1}\right) / 4>0, k=\left[\left(b_{2}-b_{1}\right)+\left(2\left(b_{1}{ }^{2}+b_{2}{ }^{2}\right)\right)^{1 / 2}\right] /\left(b_{1}+b_{2}\right)$. We observe that the $x_{2}$-coordinate must satisfy, by $(3.1)$, the equation of motion

$$
\ddot{x}+a x+b x^{3}=0,
$$

Duffing's equation, with $a=a_{2}-a_{3}(k-1), b=b_{2}-b_{3}(k-1)^{3}$. The desired solution of this equation is [7]:

$$
\begin{gathered}
x=x_{0} c n\left(u, \lambda^{2}\right), \text { where } \\
t=\left(a+b x_{0}^{2}\right)^{-1 / 2} u, \quad u=\int_{0}^{\Phi}\left(1-\lambda^{2} \sin ^{2} \psi\right)^{-1 / 2} d \psi, \\
\lambda^{2}=\frac{1}{2} b x_{0}^{2}\left(a+b x_{0}^{2}\right)^{-1}, \quad c n\left(u, \lambda^{2}\right)=\cos \varphi .
\end{gathered}
$$

At this point we leave $k$ indicated, rather than explicitly substitute. Since $\dot{x}(0)=0$ by choice, we can insert $x_{2}=x_{0}, x_{1}=k x_{0}$ in the Hamiltonian (1.1) at energy $h$, recall that $x_{0}^{2}$ $\geq 0$ and solve to obtain

$$
x_{0}{ }^{2}=\frac{\left(\left(a_{1} k^{2}+a_{2}+a_{3}(k-1)^{2}\right)^{2}+4 h\left(b_{1} k^{4}+b_{2}+b_{3}(k-1)^{4}\right)\right)^{1 / 2}-\left(a_{1} k^{2}+a_{2}+a_{3}(k-1)^{2}\right)}{b_{1} k^{4}+b_{2}+b_{3}(k-1)^{4}} .
$$

The next step involves computing the variational equations along the mode. One then performs the change of variables of Theorem 2.1 , specifically

$$
\left(1+k^{2}\right)^{1 / 2} U_{1}=k z_{1}+z_{2}, \quad\left(1+k^{2}\right)^{1 / 2} U_{2}=-z_{1}+k z_{2} .
$$

To see that this is indeed one of the coordinate changes whose existence was discussed in Theorem 2.1 we first note that, since $\eta=1$, the similarity constants for the in- and out-ofphase modes are $k$ and $-1 / k$. One then finds that the constant $K$ must satisfy $K=k-1 /$ $k$. Using this in the recipe for $a, b, c, d$ in Theorem 2.1 yields the above transformation. With this and a change of independent variable from $t$ to $u$ (see (4.1)) we obtain, after a fair amount of algebra:

$$
\begin{aligned}
U_{1}^{\prime \prime} & +\left[4 \lambda^{2}+1-2 \cdot 3 \lambda^{2} s n^{2}\left(u, \lambda^{2}\right)\right] U_{1}=0 \\
U_{2}^{\prime \prime} & +\left[\left(a_{1}+a_{3}(k+1)+3\left(b_{1} k^{2}+b_{3}(k+1)(k-1)^{2}\right) x_{0}^{2}\right) /\left(a+b x_{0}{ }^{2}\right)\right. \\
& \left.-3 \cdot 2\left[\left(b_{1} k^{2}+b_{3}(k+1)(k-1)^{2}\right) /\left(b_{2}-b_{3}(k-1)^{3}\right)\right] \lambda^{2} s n^{2}\left(u, \lambda^{2}\right)\right] U_{2}=0,
\end{aligned}
$$


two Lamé equations. The first equation has the periodic solution $\operatorname{sn}\left(u, \lambda^{2}\right) \operatorname{dn}\left(u, \lambda^{2}\right)$ and so if $U_{1}$ and $U_{2}$ are as in Theorem 2.6, we conclude that $U_{1}(2 \mathscr{K})=1$ and $U_{1}{ }^{2}(\mathscr{K})=1$, where $\mathscr{K}=\mathscr{K}\left(\lambda^{2}\right)$ is the real half period of the Jacobi elliptic function $\operatorname{sn}\left(u, \lambda^{2}\right)$. In view of the results in Sec. 2, we are now left with computing $U_{2}(K)$ as a function of the various parameters of the Hamiltonian (1.1). The equation for $U_{2}$ has the form

$$
U_{2}^{\prime \prime}+\left[A-6 \beta \lambda^{2} s n^{2}\left(u, \lambda^{2}\right)\right] U_{2}=0,
$$

with $A$ and $\beta$ the obvious expressions. Letting $\sigma=b_{1} / b_{2}$ and using the formulae for $b_{3}$ and $k$, we compute

$$
\beta=\beta(\sigma)=\left[3(\sigma+1)^{2}+2(\sigma+1)\left(2\left(\sigma^{2}+1\right)\right)^{1 / 2}\right] /\left(\sigma^{2}+18 \sigma+1\right) .
$$

For $\sigma=1$ (the outer nonlinearities are the same), $\beta(1)=1$. As $\sigma \rightarrow 0$ (the nonlinearity on the right becomes much larger than the one on the left), $\beta(\sigma) \rightarrow 3+2 \sqrt{ } 2$. It is well known [12] that the Lamé equation

$$
y^{\prime \prime}+\left(A-B \lambda^{2} s n^{2}\left(u, \lambda^{2}\right)\right) y=0
$$

has exactly $n$ bounded intervals of instability when $B=n(n+1)$ and has no collapsing intervals of instability if $B \neq n(n+1)$ ( $n$ a positive integer). Since $B=B(\sigma)=3 \cdot 2 \beta(\sigma)$, we have

$$
\begin{array}{ll}
\sigma=1, & B(\sigma)=2 \cdot 3, \\
\sigma=1 / 7, & B(\sigma)=3 \cdot 4, \\
\sigma=\left[729-40(329)^{1 / 2} / 71 \cong .0488,\right. & B(\sigma)=4 \cdot 5, \\
\sigma=\left[40+41 \sqrt{2}-(4958+3280 \sqrt{ } 2)^{1 / 2}\right] / 2 \cong .0102, & B(\sigma)=5 \cdot 6,
\end{array}
$$

and no other possibilities arise: all other choices of nonlinearity ratios lead to equations with no collapsing intervals of instability. To examine the stability properties of the inphase mode, we must determine the exact stability characteristics of this Lamé equation for various choices of the parameters. To do this we must study the coefficient $A$, which we can easily see is of the form $A=A\left(a_{1}, a_{2}, b_{1}, b_{2}, h\right)$. We observe that

$$
\begin{aligned}
\lim _{h ! 0} A\left(a_{1}, a_{2}, b_{1}, b_{2}, h\right) & =\left[a_{1}+a_{3}(k+1)\right] /\left[a_{2}-a_{3}(k-1)\right] \\
= & {\left[2(\tau-\sigma)+(\tau-1)\left(2\left(\sigma^{2}+1\right)\right)^{1 / 2}\right] /\left[2(\tau-\sigma)-(\tau-1)\left(2\left(\sigma^{2}+1\right)\right)^{1 / 2}\right], }
\end{aligned}
$$

with $\tau=a_{2} / a_{1}>1$ and $\sigma=b_{1} / b_{2}<1$. This limit can be made arbitrarily large by first choosing $\tau$ large and then choosing $\sigma$ close to 1 . We can also show

$$
\lim _{h \downarrow 0} \lambda^{2}=0 ; \quad \lim _{h \uparrow \infty} \lambda^{2}=1 / 2
$$

$\lim _{h \uparrow \infty} A\left(a_{1}, a_{2}, b_{1}, b_{2}, h\right)=3\left[b_{1} k^{2}+b_{3}(k+1)(k-1)^{2}\right] /\left[b_{2}-b_{3}(k-1)^{3}\right] \equiv \delta(\sigma) ;$

$$
\lim _{\sigma \uparrow 1} \delta(\sigma)=3 \text {. }
$$

We summarize the results above in the following lemma.

LEMMA 4.1. Given any positive integer $n$ and a small positive number $\omega$, there exists two 
numbers $M>1,0<m<1$, such that if $a_{2} / a_{1}>M$ and $1-m<b_{1} / b_{2}<1$, then

$$
A\left(a_{1}, a_{2}, b_{1}, b_{2}, 0\right)>n^{2}, \quad \lim _{h \uparrow \infty} A\left(a_{1}, a_{2}, b_{1}, b_{2}, h\right)<3+\omega .
$$

For the reader's convenience we include two figures (Figs. 3 and 4) representing the stability chart for the Lamé equation when $B=6$ and when $B>6$ but near 6 (the latter one will be only indicated, and is only qualitatively valid). Note that the stability boundaries enclosing nontrivial regions of instability correspond to unstable solutions. The curves joining the $A$-axis to the point $(1 / 2,3)$ or $(1 / 2,3 \pm)$ represent images on the $A-\lambda^{2}$ plane for the curves given by $A=A\left(a_{1}, a_{2}, b_{1}, b_{2}, h\right)$ as $h$ goes from 0 to $+\infty$. The case $B=6$ was studied in detail in [11], and we refer the reader to that paper. The case in which $B$ is slightly greater than 6 will now be sketched: the continuity of solutions with respect to parameters implies that the "instability boundaries" for $B$ close to 6 are near those for $B=6$. Thus Fig. 4 is at least qualitatively true, except that we do not know that the point $(1 / 2, A$ $\left.\left(a_{1}, a_{2}, b_{1}, b_{2}, \infty\right)\right)$ lies exactly on an "instability boundary" (which is the case for $B=6$ and the symmetric oscillator: in that case $\left.A=A\left(a_{1}, a_{3}, h\right)\right)$. The "stability regions" are foliated by analytic "constant discriminant" curves (curves where the discriminant of the Lamé equation is constant) and these are constant eigenvalue curves for the linearized Poincaré map. As $h$ increases, with the other parameters fixed, the point $\left(\lambda^{2}(h), A(h)\right)$ moves down the curve of Fig. 4 in a smooth manner. This variation is smooth enough, so that, if $H$ is the set of "good" eigenvalues defined in connection with Theorem 1.1, then the set of $h$ for which the corresponding eigenvalue is unimodular but not in $H$ has measure 0 . Thus by Theorem 1.1 we have:

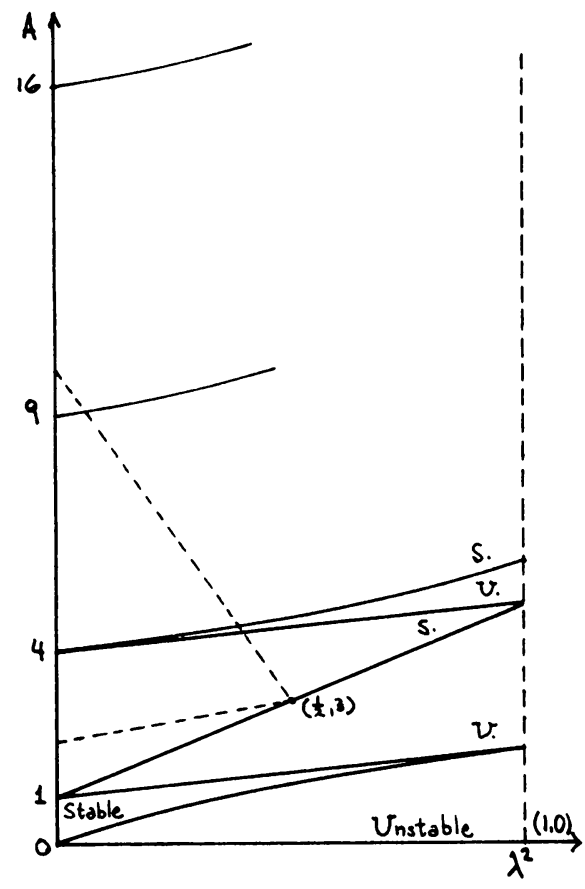

Fig. 3.

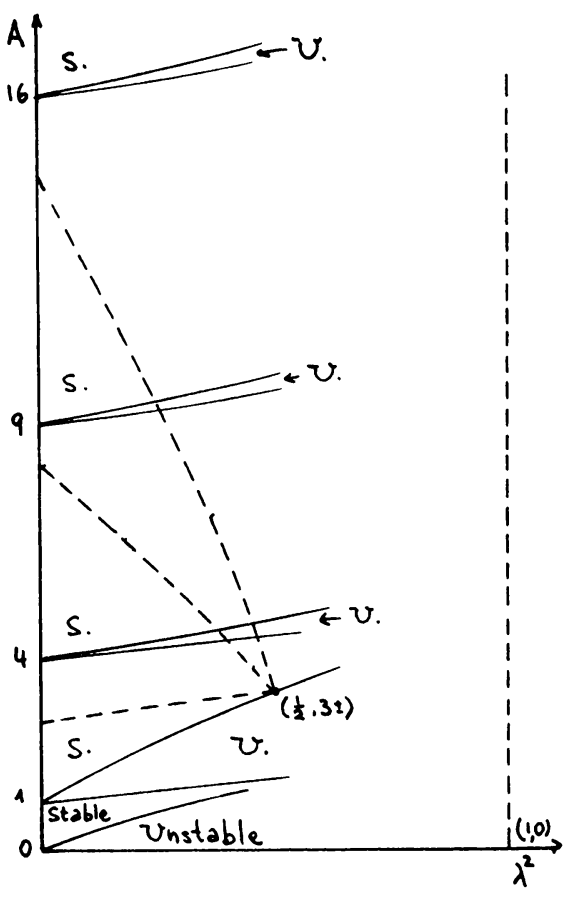

FIG. 4. 
THEOREM 4.2. Consider the in-phase mode $x_{1}=k x_{2}$ of the nonlinear oscillator (1.1) with

$$
\begin{aligned}
\alpha_{1} & =\alpha_{2}=1 ; \quad a_{2}>a_{1}>0 ; \quad b_{2}>b_{1}>0 ; \\
a_{3} & =\left[\left(a_{2}-a_{1}\right)\left(b_{2}+b_{1}\right)\right] / 2\left(b_{2}-b_{1}\right) ; \quad b_{3}=\left(b_{2}+b_{1}\right) / 4 ; \\
k & =\left[\left(b_{2}-b_{1}\right)+\left(2\left(b_{1}{ }^{2}+b_{2}{ }^{2}\right)\right)^{1 / 2}\right] /\left(b_{1}+b_{2}\right) .
\end{aligned}
$$

For any given positive integer $n$ there exists numbers $M$ and $m$, both positive, such that for all $a_{2} / a_{1}>M$ and $1-m<b_{1} / b_{2}<1$, the in-phase-mode will be hyperbolic over at least $n$ nontrivial intervals for the parameter $h \in[0, \infty]$. In the complement of these intervals the orbit will be elliptic stable for almost all values of $h$.

We omit the formal details of the proof; the reader is referred to [15] for the details in a simpler application. Although we cannot at present prove it, we conjecture that the inphase mode is elliptic for all large values of $h$ : this was indeed the case for the symmetric oscillator [15].

We leave the investigation of the out-of-phase modes to the interested reader, with the caveat that the computations are likely to be even messier.

5. A stronger form of stability. In the case of normal modes of "symmetric cubic" oscillators, the almost everywhere iso-energetic stability which one obtains by using the Arnold-Moser-Rüssmann criterion as in [15] or in the last section, can be considerably strengthened. To do this we must analyse the reduced Poincaré map $P$ associated with a normal mode $\omega(t)$. Here, unlike the situation of the preceding sections, we shall need more than just the linear part of the map.

Let $h$ be the energy of $\omega(t)$ and let $\Sigma_{h}$ denote the corresponding energy surface defined by $H=h$ where $H$ is the Hamiltonian of our system. In $\Sigma_{h}$ we choose a small 2-disk $\sigma$ orthogonal to $\dot{\omega}(0)$ at $\omega(0)$. If $x$ is a point of $\sigma$ which is sufficiently near $\omega(0)$ then the positive orbit through $x$ will intersect $\sigma$ at least once. The point $P(x)$ of first return defines the reduced Poincaré map $P$. By choosing suitable coordinates for $\sigma$, with $\omega(0)$ corresponding to the origin, we may regard $P$ as a map from a neighborhood of the origin in $R^{2}$ to $R^{2}$ which has the origin as a fixed point.

The map $P$ is real analytic at the origin and therefore has a power series expansion which converges in a disk centered at $x=0, y=0$, say:

$$
x^{\prime}=f_{1}(x, y)+f_{2}(x, y)+\cdots, \quad y^{\prime}=g_{1}(x, y)+g_{2}(x, y)+\cdots
$$

where the $f_{i}, g_{i}$ are homogeneous polynomials of degree $i$. Now, as noted earlier, the eigenvalues $\lambda \pm$ of the linear part of $P$ have product 1. (This also follows from the area-preserving character of $P$.)

In [22] it is shown that if the eigenvalues have unit modulus (i.e., if $P$ is not hyperbolic) and if $\lambda^{k} \neq 1$ for $k=1,2,3,4$, then there is a real analytic area preserving change of variables which takes $P$ into so-called Birkhoff normal form through terms of order 3 . If the change of variables is denoted by $(x, y)=C((\xi, \eta))$ then the normal form $N=C^{-1} P C$ may be written very simply in complex notation as:

$$
\zeta^{\prime}=\lambda \zeta \exp \left(i \gamma|\zeta|^{2}\right)+O_{4}
$$

where $\zeta=\xi+i \eta, \gamma$ is real, and $O_{4}$ denotes a convergent power series in $\zeta, \xi$ beginning with terms of order 4 .

The Twist Theorem of Kolmogorov, Arnold and Moser [1], [13], then states that if the 
coefficient $\gamma$ in the power series for $\omega$ is not zero, then the origin is a stable fixed point for $P$. In our context, this means that if $\gamma \neq 0$ then our normal mode will be iso-energetically stable. Now $\gamma$ may be regarded as a function of the parameters appearing in $H$, e.g., the $a_{i}$ and $b_{i}$ of formula (1.1), together with the energy $h$, and we wish to determine the nature of the set of parameters where $\gamma \neq 0$. Actually, it follows from the statement of the Twist Theorem which is given in [13] that if $\gamma$ is not zero at some point $\left(a_{i}, b_{i}, h\right)$ in parameter space, then the orbit is not only iso-energetically stable but also orbitally stable in the full four-dimensional phase space.

Thus our task is to compute the form (5.1) well enough so that the twist coefficient $\gamma$ can be analyzed as a function of the parameters $a_{i}, b_{i}$ and $h$. We indicate how these computations go for the in-phase mode of the symmetric, cubic oscillator

$$
H=\frac{1}{2}\left(y_{1}{ }^{2}+y_{2}{ }^{2}\right)-a_{2} x_{1} x_{2}+\frac{1}{2}\left(a_{1}+a_{2}\right)\left(x_{1}{ }^{2}+x_{2}{ }^{2}\right)+\frac{b_{1}}{4}\left(x_{1}{ }^{4}+x_{2}{ }^{4}\right)+\frac{b_{2}}{4}\left(x_{1}-x_{2}\right)^{4}
$$

where $a_{i}, b_{i}>0\left(a_{1}, b_{1}\right.$ are the constants of the outer springs, $a_{2}$ and $b_{2}$ the constants of the middle spring).

We begin by making the substitutions

$$
\left[\begin{array}{c}
x_{1} \\
x_{2} \\
y_{1} \\
y_{2}
\end{array}\right]=1 / \sqrt{ } 2\left[\begin{array}{ccll}
1 / \sqrt{ } \alpha_{1} & 1 / \sqrt{ } \alpha_{2} & 0 & 0 \\
1 / \sqrt{ } \alpha_{1} & 1 /-\sqrt{ } \alpha_{2} & 0 & 0 \\
0 & 0 & \sqrt{ } \alpha_{1} & \sqrt{ } \alpha_{2} \\
0 & 0 & \sqrt{ } \alpha_{1} & -\sqrt{ } \alpha_{2}
\end{array}\right]\left[\begin{array}{c}
q_{1} \\
q_{2} \\
p_{1} \\
p_{2}
\end{array}\right]
$$

where $\alpha_{1}=\sqrt{ } a_{1}$ and $\alpha_{2}=\left(a_{1}+2 a_{2}\right)^{1 / 2}$ followed by the partial action-angle substitution:

$$
p_{1}=(2 R)^{1 / 2} \sin \tau, \quad q_{1}=(2 R)^{1 / 2} \cos \tau, \quad q_{2}=x, \quad p_{2}=y .
$$

The total transformation is canonical and brings the Hamiltonian (5.3) into the form:

$$
H=\alpha_{1} R+\frac{\alpha_{2}}{2}\left(x^{2}+y^{2}\right)+h_{0} x^{4}+h_{1} x^{2} R+h_{2} R^{2}
$$

where $h_{0}=\left(b_{1}+8 b_{2}\right) / 8 \alpha_{2}{ }^{2}, h_{1}=3 b_{1} \cos ^{2} \tau / 2 \alpha_{1} \alpha_{2}, h_{2}=b_{1} \cos ^{4} \tau / 2 \alpha_{1}{ }^{2}$. In these coordinates the in-phase mode $\omega(t)$ is given by $x(t) \equiv y(t) \equiv 0$.

We now fix any energy $h>0$. The equation $H=h$, which defines the corresponding invariant three-dimensional energy surface $\Sigma_{h}$ may be solved for $R$ as a function of $x, y, \tau$ (and the parameters $a_{i}, b_{i}, h$ ). Indeed, for $|x|,|y|$ sufficiently small we may write $R$ as a power series in $x$ and $y$ involving only even powers of these variables:

$$
R=\Phi(x, y)=\sum \varphi_{i j} x^{i} y^{j}\left(\begin{array}{c}
i, j \text { even } \\
0 \leq i, j
\end{array}\right)
$$

We shall need the first six coefficients in what follows. They can be found using (5.4) and (5.5) as functions of the $h_{i}, a_{i}, b_{i}, h$ and $\varphi_{02}, \varphi_{20}$ (see Appendix).

Since $d \tau / d t \neq 0$, we may replace $t$ by $\tau$ as the new time variable and then the differential equations may be expressed near the in-phase mode in $\Sigma_{h}$ by:

$$
\dot{x}=\partial \Phi / \partial y, \quad \dot{y}=-\partial \Phi / \partial x
$$

where the dot denotes differentiation with respect to $\tau$. With $\tau$ as time variable, the Poin- 
caré map will be obtained by computing the general solution and then following a point from time $\tau=0$ to time $\tau=2 \pi$. We now write out the general solution $P(\tau)$ as a power series in the initial values $x=x(0)$ and $y=y(0)$, valid for $|x|,|y|$ small:

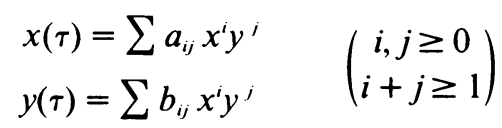

where the $a_{i j}, b_{i j}$ are functions of $\tau$ and the parameters $a_{i}, b_{i}$ in the original Hamiltonian. In order to compute the twist coefficient $\gamma$ we need information about the coefficients $a_{i j}$, $b_{i j}$ for $i+j \leq 3$. Substituting (5.7) into (5.6) and equating coefficients, we find first that

$$
P_{1}(\tau) \equiv\left(\begin{array}{ll}
a_{10}(\tau) & a_{01}(\tau) \\
b_{10}(\tau) & b_{01}(\tau)
\end{array}\right)
$$

is just the principal matrix solution of the equation:

$$
\dot{Z}=A Z, \text { where } A=\left(\begin{array}{cc}
0 & 2 \varphi_{02} \\
-2 \varphi_{20} & 0
\end{array}\right) \text {. }
$$

(This, of course, also follows from the fact that (5.8) is the linear part of the Hamiltonian system (5.6) and that $P_{1}(\tau)$ is the linear part of the general solution $P(\tau)$.) Next we observe that the second-order coefficients, taken in pairs $\left(a_{20}, b_{20}\right)$, etc., satisfy Eq. (5.8) with initial data $(0,0)$. Hence all six second-order coefficients vanish identically.

We now need to compute the third-order coefficients in (5.7). Fortunately they can be expressed in closed form as integrals of polynomial expressions in the lower order $a_{i j}, b_{i j}$ and the $\varphi_{i j}$ 's with $i+j \leq 4$, once again we refer to the Appendix for details. The Poincaré map $P$ is then simply $P(2 \pi)$.

Now recall that to put $P$ in Birkhoff normal form through terms of order three, the eigenvalues $\lambda_{ \pm}$of the linear part must have unit modulus and satisfy $\lambda^{k} \neq 1$ for $k=1,2,3$, 4. The eigenvalues are just $\Delta \pm\left(\Delta^{2}-4\right)^{1 / 2} / 2$ where $\Delta=\left(a_{10}(2 \pi)+b_{01}(2 \pi)\right) / 2$ in the notation of (5.7). The conditions $\Delta^{2}<4, \Delta \neq 0,-1$ imply the above conditions on $\lambda$. Therefore we consider only parameter values $\left(a_{1}, a_{2}, b_{1}, b_{2}, h\right)$ for which these conditions hold; let $\mathfrak{A}$ denote this set of "admissible" parameter values.

If one now follows the transformation into normal form described in [12, page 155] one finds that $\gamma$ may be computed entirely in terms of the first-, second-, and third-order coefficients, $a_{i j}$ and $b_{i j}$. After a good deal of work (see the Appendix), we get a formula for $\gamma$ which looks like this:

$$
\begin{aligned}
\gamma= & -2 \int_{0}^{2 \pi}\left\{\varphi_{40}(s) G\left(a_{10}(s), a_{01}(s), a_{10}(s), a_{01}(s)\right)\right. \\
& \left.+\varphi_{22}(s) G\left(a_{10}(s), a_{01}(s), b_{10}(s), b_{01}(s)\right)+\varphi_{04}(s) G\left(b_{10}(s), b_{01}(s), b_{10}(s) b_{01}(s)\right)\right\} d s
\end{aligned}
$$

where $G$ is a certain homogeneous polynomial of degree 4 .

Regarding $\gamma$ as a function of parameter points in the set $\mathfrak{A} \subset\left(R^{5}\right)^{+}$, we observe that it is real analytic at each point of $\mathfrak{A}$. (Actually for the in-phase mode, it is enough to observe that $\gamma$ is $C^{1}$ in all variables; however, in other cases analyticity becomes important.)

Finally, if one computes $\partial \gamma / \partial b_{2}$ one finds that it is everywhere positive in $\mathfrak{A}$. Consequently the equation $\gamma=0$ defines a set which is a four-dimensional manifold $\mathfrak{R}$ lying in $\mathfrak{A}$. Of more interest perhaps is the fact that $\mathfrak{N}$ will be a closed set of measure 0 in $\left(R^{5}\right)^{+}$.

Combining this result with the Twist Theorem mentioned earlier, we have: 
THEOREM 5.1. There is a closed manifold $\mathfrak{N}$ of codimension 1 and measure 0 in $\mathfrak{U}$ such that, for parameter values in $\mathfrak{U}-\mathfrak{N}$, the in-phase mode of $(5.1)$ is stable in phase space.

Note that $\mathfrak{A}$ can be regarded as the set $\mathfrak{E}$ of parameters where $\Delta^{2} \leq 4$ (i.e., the nonhyperbolic parameter values) minus the set $\mathfrak{B}$ where $\Delta= \pm 2,0,-1$; certainly $\mathfrak{B}$ is closed and of measure 0 . Thus whenever the mode of Theorem 5.1 is not hyperbolic (and thus

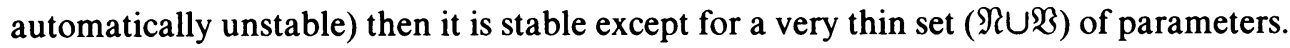

The above result holds also for the out-of-phase mode up to and including integral formula (5.9) for $\gamma$. However, at present we cannot find an everywhere nonvanishing partial derivative of $\gamma$ and thus are unable to prove that the set $\mathfrak{R}$ is a manifold. Since $\mathfrak{N}$ is the zero set of a continuous function it is automatically closed. It can also be shown that $\Re$ has measure 0 as follows. If $\mathfrak{N}$ had positive measure then $\gamma$ would vanish identically on some connected component of the open set $\mathfrak{U}$. (This can be proved using Fubini's theorem and the analyticity of $\gamma$.) Thus, we wish to show that each component of $\mathfrak{U}$ contains $a$ point where $\gamma$ does not vanish.

This may be seen by the following argument (which will apply in much more generality than it appears here). Let $p=\left(a_{1}, a_{2}, b_{1}, b_{2}, h\right)$ be a point in $\mathfrak{U}$; thus $\Delta^{2}(p)<4$. Let $\mathfrak{S}$ be the subspace of $\left(R^{5}\right)^{+}$consisting of all points whose $a_{1}, b_{1}, b_{2}$ coordinates agree with those of $p$; thus, $\mathfrak{S}$ is coordinatized by $a_{2}$ and $h$. Let $S(p)$ be the connected component of the constant discriminant set $\Delta=\Delta(p)$ which contains $p$; it is natural to refer to $S(p)$ as the constant discriminant hypersurface containing $p$. What one can show is that the set $\mathscr{U} \cap \mathfrak{S}$ is foliated by the constant discriminant curves $\{S(p) \cap \mathfrak{S} \mid p \epsilon \mathfrak{U}\}$ and that these $S(p) \cap \mathfrak{S}$ are indeed curves which abut the $a_{2}$-axis given by $h=0$. Moreover, $\mathfrak{U} \cap \mathfrak{S}$ will contain $a$ neighborhood of all but a finite number of points on the $a_{2}$-axis. The situation is pictured schematically in Fig. 5, where the lines separating components of $\mathfrak{U} \cap \mathfrak{S C}$ are the sets where $\Delta= \pm 2,0,1$.

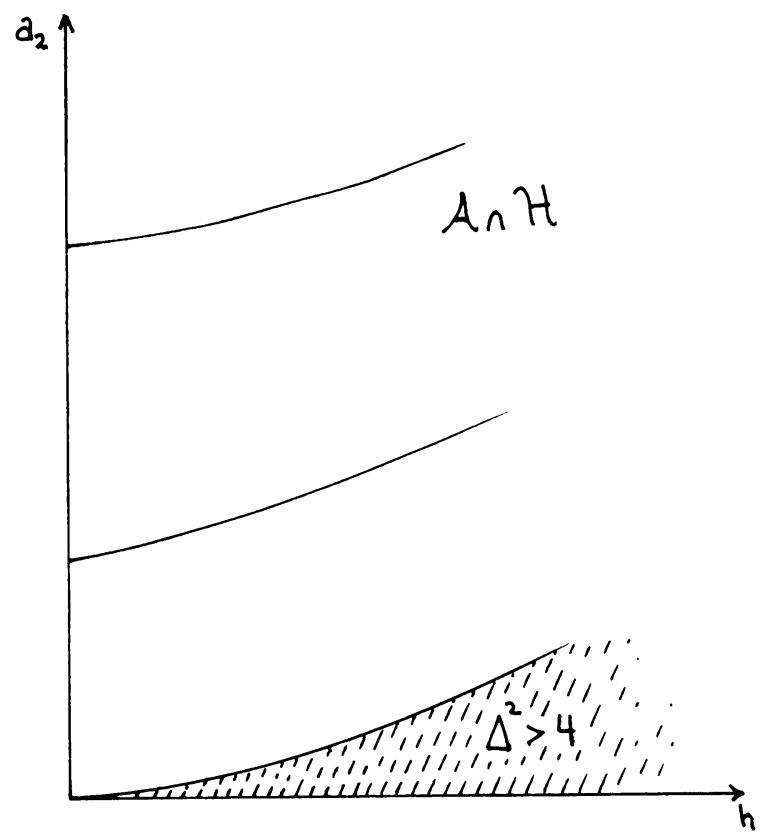

FIG. 5. 
All this follows from an analysis of how the Lamé equation governing the eigenvalues depends on the variables $a_{2}$ and $h$. This Lamé equation appears as Eq. (14) in [11]. If we write it in the form $y^{\prime \prime}+\left(\alpha+\beta s n^{2} u\right) y-0$ then the map which sends $\left(h, a_{2}\right)$ to $(\beta, \alpha)$ can be shown to be a diffeomorphism of $\mathfrak{U} \cap \mathfrak{S}$ onto an open subset of the first stability region.

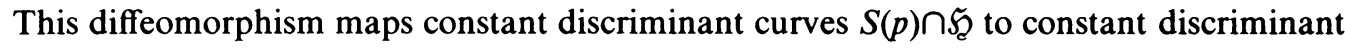
curves of the Lamé equation. The above assertions concerning $\mathfrak{U} \cap S \mathrm{~S}$ follow from the geometry of the stability regions and constant discriminant curves for the Lamé equation.

Now, in [3] it was shown that, for fixed $a_{1}, b_{1}, b_{2}>0$, there is a finite set $F$ such that if $a_{2} \notin F$ and $h$ is sufficiently small then the twist associated with the out-of-phase mode is not zero. By what we just proved, each component of $\mathfrak{U}$ will certainly contain a point with $a_{2} \notin F$ and $h$ sufficiently small, so we are done.

(Remark. A weaker result than the above was stated in [3] but the same proof actually gives the above assertion, since-as was alluded to in the middle of p. 41 - one merely needs a finite number of conditions on the $\alpha_{1}$ and $\alpha_{2}$ of that paper. We also wish to correct a mistake in [3] at this point. D. Rod has pointed out that there is a gap in going from formula (9) to formula (11). However, the formula for $\gamma$ is still correct. This can be seen by beginning with (9), changing to Cartesian coordinates, and then following the procedure of [22, pp. 155-173] for computing $\gamma$ as we have done in this paper.)

In summary, we have a result much like Theorem 5.1.

THEOREM 5.2. There is a closed set $\mathfrak{N}$ of measure 0 in $\mathfrak{U}$ such that for parameter values in $\mathfrak{U}-\mathfrak{N}$, the out-of-phase mode of $(5.1)$ is stable in phase space.

All of the above also applies to these modes for a general odd power restoring force up to the assertion that each component of $\mathfrak{U}$ contains a point where the twist does not vanish. Although we believe this to be the case, we do not at present know how to prove it; the governing Hill's equation is no longer a Lamé equation and, what is worse, we are unable to determine the precise connection between its constant discriminant curves and the surfaces $S(p), p \in \mathfrak{U}$.

Finally, we remark that the methods of this section apply to some non-symmetric oscillators. However, for geometrical reasons they cannot be applied to the general cubic non-symmetric case, the difficulty being that we cannot canonically transform into a form sufficiently like (5.4) to carry out the rest of the analysis as it is here developed. Thus, for these oscillators, the best stability techniques available at present are those of Sec. 2, 3 and 4 -namely decoupling, reduction, and the Arnold-Moser-Rüssmann criterion.

6. Appendix. In this section we provide some of the details omitted in Sec. 5 .

With $\alpha_{i}, h$ and $h_{i}$ as in (5.4) we first let $C=\alpha_{1}{ }^{2}+4 h h_{2}$. Then the lower-order $\varphi_{i j}$ are given by

$$
\begin{aligned}
& \varphi_{00}=\sqrt{ } C-\alpha_{1} / 2 h_{2}, \quad \varphi_{20}=\frac{h_{1} \alpha_{1}-h_{2} \alpha_{2}-h_{1} \sqrt{ } C}{2 h_{2} \sqrt{ } C}, \\
& \varphi_{02}=-\alpha_{2} / 2 \sqrt{ } C, \quad \varphi_{40}=-\frac{h_{0}-h_{1} \varphi_{20}-h_{2} \varphi_{20}^{2}}{\sqrt{ } C}, \\
& \varphi_{22}=-\frac{h_{1} \varphi_{02}-2 \varphi_{20} \varphi_{02}}{\sqrt{ } C}, \quad \varphi_{04}=-h_{1} \varphi_{02}^{2} / \sqrt{ } C .
\end{aligned}
$$

The next step is to compute the $a_{i j}$ and $b_{i j}$ of formula (5.7) where $i+j=3$. One 
finds by substitution of (5.7) into (5.6) that these functions satisfy

$$
\left[\begin{array}{l}
a_{i j} \\
b_{i j}
\end{array}\right]=A\left[\begin{array}{l}
a_{i j} \\
b_{i j}
\end{array}\right]+\left[\begin{array}{l}
e_{i j} \\
f_{i j}
\end{array}\right]
$$

with initial data $a_{i j}(0)=0=b_{i j}(0)$, where the forcing functions $e_{i j}, f_{i j}$ are given by:

$$
\begin{aligned}
& e_{30}=4 \varphi_{04} b_{10}{ }^{3}+2 \varphi_{22} a_{10}{ }^{2} b_{10} \\
& f_{30}=-4 \varphi_{04} a_{10}{ }^{3}-2 \varphi_{22} a_{10} b_{10}{ }^{2} \\
& e_{21}=4 \varphi_{04}\left(3 b_{10}{ }^{2} b_{01}\right)+2 \varphi_{22}\left(a_{10}{ }^{2} b_{01}+2 a_{10} a_{01} b_{10}\right) \\
& f_{21}=-4 \varphi_{40}\left(3 a_{10}{ }^{2} a_{01}\right)-2 \varphi_{22}\left(a_{01} b_{10}{ }^{2}+2 a_{10} b_{10} b_{01}\right) \\
& e_{12}=4 \varphi_{04}\left(3 b_{10} b_{01}{ }^{2}\right)+2 \varphi_{22}\left(a_{01}{ }^{2} b_{10}+2 a_{10} a_{01} b_{01}\right) \\
& f_{12}=-4 \varphi_{40}\left(3 a_{10} a_{01}{ }^{2}\right)-2 \varphi_{22}\left(a_{10} b_{01}{ }^{2}+2 a_{01} b_{10} b_{01}\right) \\
& e_{03}=4 \varphi_{04}\left(b_{01}{ }^{3}\right)+2 \varphi_{22}\left(a_{01}{ }^{2} b_{01}\right), \quad f_{03}=-4 \varphi_{40}\left(a_{01}{ }^{3}\right)-2 \varphi_{42}\left(a_{01} b_{01}{ }^{2}\right) .
\end{aligned}
$$

Explicit solutions of the system (6.2)-(6.3) can be written down by variation of parameters. Indeed, we have

$$
\left[\begin{array}{l}
a_{i j}(\tau) \\
b_{i j}(\tau)
\end{array}\right]=P(\tau) \int_{0}^{\tau} P^{-1}(\sigma)\left[\begin{array}{l}
e_{i j}(\sigma) \\
f_{i j}(\sigma)
\end{array}\right] d \sigma
$$

Define functions $v_{i j}, w_{i j}$ by the formulas

$$
\begin{aligned}
& v_{i j}(\tau)=\int_{0}^{\tau}\left(b_{01}(\sigma) e_{i j}(\sigma)-a_{01}(\sigma) f_{i j}(\sigma)\right) d \sigma \\
& w_{i j}(\tau)=\int_{0}^{\tau}\left(-b_{10}(\sigma) e_{i j}(\sigma)+a_{01}(\sigma) f_{i j}(\sigma)\right) d \sigma .
\end{aligned}
$$

Then the third order coefficients take the form:

$$
\begin{aligned}
& a_{i j}(\tau)=a_{10}(\tau) v_{i j}(\tau)+a_{01}(\tau) w_{i j}(\tau), \\
& b_{i j}(\tau)=b_{10}(\tau) v_{i j}(\tau)+b_{01}(\tau) w_{i j}(\tau)
\end{aligned}
$$

The coefficients of $P$ are then obtained by evaluating the $a_{i j}, b_{i j}$ at $\tau=2 \pi$.

Next we outline how to obtain the integral formula (5.9) for $\gamma$. These computations are based on [22, pp. 155-1973]. For notational convenience we shall write a, b, c, d rather than $a_{10}(2 \pi), a_{01}(2 \pi), b_{10}(2 \pi), b_{01}(2 \pi)$. We now assume that the parameter point $p$ $=\left(a_{1}, a_{2}, b_{1}, b_{2}, h\right)$ lies in $\mathfrak{A}$. In particular, $\Delta^{2}<2$ and this implies $b \neq 0$. We let $\lambda$ be that eigenvalue of $P$ satisfying $\operatorname{sgn}(\operatorname{Im} \lambda)=-\operatorname{sgn}(b)$.

To compute $\gamma$ we begin with $P$ in form (5.1) and transform into the normal form (5.2). We first complexify by the substitution $x=\alpha \xi+\bar{\alpha} \eta, y=\beta \xi+\bar{\beta} \eta$ where $\alpha$ and $\beta$ are chosen so that $\alpha \bar{\beta}-\beta \bar{\alpha}=i / 2$ and $a \alpha+b \beta=\alpha \lambda, c \alpha+d \beta=\beta \lambda, \lambda$ having been chosen just above. To be specific we shall take $\alpha$ to be the positive real number satis- 
fying $\alpha^{2}=-b / 4 \operatorname{Im} \lambda$ and $\beta=\alpha(\lambda-a) / b$. If we denote the above linear transformation by $C_{0}$ then $C_{0}^{-1} P C_{0} \equiv S$ has the form:

$$
\xi^{\prime}=\lambda \xi+p_{2}(\xi, \eta)+p_{3}(\xi, \eta)+\cdots, \quad \eta^{\prime}=\lambda \eta+q_{2}(\xi, \eta)+q_{3}(\xi, \eta)+\cdots
$$

where the $p_{i}, q_{i}$ are homogeneous polynomials of degree $i$ and $\bar{p}_{i}(\xi, \eta)=q_{i}(\xi, \eta)$, where the bar means conjugation of coefficients. (Note: In our case $p_{2} \equiv q_{2} \equiv 0$.) If one now follows the transformation of (6.7) into Birkhoff normal form through terms of order 4 as described in pages 155-173 of [22], then one finds that the twist coefficient $\gamma$ of order 2 satisfies an equation of the form

$$
\lambda_{i} \gamma=p_{21}+\delta
$$

where, in general, $\delta$ is a rather messy expression involving the second-order $p_{2}$ and $q_{2}$. In our case this expression is automatically 0 since the second-order $f_{i}, g_{i}$ in (5.1) are zero.

Thus we need $p_{21}$ which is, of course, the coefficient of $\xi^{2} \eta$ in $p_{3}$. From the form of $C_{0}$ we find that $p_{21}$ is $2 / i$ times the quantity $\left(\bar{\beta}_{30}-\alpha g_{30}\right)\left(3 \alpha^{3}\right)+\left(\bar{\beta} f_{21}-\alpha g_{21}\right)\left(\alpha^{2} \bar{\beta}+\right.$ $\left.2 \alpha^{2} \beta\right)+\left(\bar{\beta} f_{21}-\alpha g_{21}\right)\left(2 \alpha \beta \bar{\beta}+\alpha \beta^{2}\right)+\left(\bar{\beta} f_{03}-\alpha g_{03}\right)\left(3 \beta^{2} \bar{\beta}\right)$, where it should be recalled that $\alpha$ was taken real. The $f_{i j}, g_{i j}$ are simply the $a_{i j}, b_{i j}$ of (6.6) evaluated at $\tau=2 \pi$. Note also the relations $\bar{\beta} a-\alpha c=\lambda \bar{\beta}$ and $\bar{\beta} b-\alpha d=-\alpha \lambda$. Using these facts we may write the expression for $p_{21}$ as $2 \lambda / i$ times $\left.3 \alpha^{3} \bar{\beta} v_{30}-\alpha w_{30}\right)+\left(\alpha^{2} \bar{\beta}+2 \alpha^{2} \beta\right)\left(\bar{\beta} v_{21}-\alpha w_{21}\right)$ $+\left(2 \alpha \beta \bar{\beta}+\alpha \beta^{2}\right)\left(\bar{\beta} v_{12}-\alpha w_{12}\right)+\left(3 \beta^{2} \bar{\beta}\right)\left(\bar{\beta} v_{03}-\alpha w_{03}\right)$ where all the $v_{i j}, w_{i j}$ are given by (6.5) evaluated at $\tau=2 \pi$.

This expression becomes somewhat more attractive if we write it as one large integral. To this end one first needs to write out the $v_{i j}, w_{i j}$ using the expressions (6.3) for the $e_{i j}, f_{i j}$. For example, $v_{30}$ is the integral from 0 to $2 \pi$ of the expression $\varphi_{40}\left(4 a_{10}{ }^{3}\right)+$ $\varphi_{22}\left(2 a_{10}{ }^{2} b_{10} b_{01}+2 a_{10} a_{01} b_{10}{ }^{2}\right)+\varphi_{04}\left(4 b_{10}{ }^{3} b_{01}\right)$ and similar expressions appear for the other seven $v_{i j}, w_{i j}$. Then, bringing the $\alpha$ and $\beta$ inside the integral sign and collecting coefficients of the $\phi_{i j}$, we find that $p_{21}$ is $2 \lambda / i$ times the integral of formula (5.9), which we shall not rewrite here. The polynomial $G$ is given by

$$
\begin{aligned}
& G(p, q, r, s)=\left(12 \alpha^{4}\right) p^{2} r^{2}+\left(12 \alpha^{4} \Gamma\right)(p r)(p s+g r) \\
& +\left(\gamma^{2}+2 \alpha^{2} \beta \bar{\beta}\right)\left(2 p^{2} s^{2}+8 p q r s+2 q^{2} r^{2}\right)+(12 \beta \bar{\beta} \Gamma)(q s)(p s+q r)+\left(12 \beta^{2} \bar{\beta}^{2}\right) q^{2} r^{2},
\end{aligned}
$$

where $\Gamma=\alpha \bar{\beta}+\beta \bar{\alpha}$. In view of the relationship (6.8) between $\gamma$ and $p_{21}$ we obtain formula (5.9) for $\gamma$.

The computation of $\partial \gamma / \partial b_{2}$ is quite straightforward. We have $\partial h_{1} / \partial b_{2}=\partial h_{2} / \partial b_{2}=0$, whence $\partial \varphi_{00} / \partial b_{2} \equiv \partial \varphi_{20} / \partial b_{2} \equiv \partial \varphi_{02} / \partial b_{2} \equiv 0$. Therefore the partials of the $a_{i j}, b_{i j}$ with $i+$ $j=1$ also vanish identically. Next $\partial h_{0} / \partial b_{2}=8 / 8 \alpha_{2}{ }^{2}$ and we get that $\partial \varphi_{40} / \partial b_{2}=-1 /$ $\alpha_{2}{ }^{2} \sqrt{ } C$, while the partials of the other two fourth-order $\varphi_{i j}$ vanish. It follows that

$$
\frac{\partial \gamma}{\partial b_{2}}=-2 \int_{0}^{2 \pi} \frac{\partial \varphi_{40}}{\partial b_{2}}=(-2)\left(-\frac{1}{\alpha_{2}{ }^{2}}\right) \int_{0}^{2 \pi} \frac{1}{\sqrt{ } C}\left(a_{10}{ }^{2} \alpha^{2}+b_{01}{ }^{2} \beta^{2}+a_{10} a_{01} \Gamma\right)^{2}
$$

where we have suppressed the arguments of the integrands. The last expression shows that $\partial \gamma / \partial b_{2}>0$.

[1] V. I. Arnold, Kleine Nenner and Stabilitätsprobleme der klassischen Mechanik und der Himmelsmechanik, Uspekhi Mat. Nauk USSR 18, 91-192 (1963) 
[2] F. M. Arscott, Periodic differential equations, Pergamon, 1964

[3] R. Churchill, H. Jurenka and E. Thomas, A note on iso-energentic stability, Int. J. Nonlinear Mech., 12, 37-43 (1977)

[4] R. Churchill, G. Pecelli, and D. Rod, Stability transitions for periodic orbits in Hamiltonian systems, to appear

[5] R. Churchill, G. Pecelli, D. Rod and S. Sackolic, Coexistence of stable and random motion, Rocky Mountain J. Math. 7, 445-456 (1977)

[6] C. H. Cooke and R. A. Struble, On the existence of periodic solutions and normal mode vibrations of nonlinear systems, Quart. Appl. Math. 24, 177-193 (1966)

[7] W. B. Gordon, $A$ theorem on the existence of periodic solutions to Hamiltonian systems with convex potential, J. Diff. Eq. 10, 324-334 (1971)

[8] P. Hartman, Ordinary differential equations, John Wiley, 1964

[9] C. S. Hsu, On a restricted class of Hill's equations and some applications, J. Appl. Mech. 28e, 551-556 (1961).

[10] C. S. Hsu, On the parametric excitations of a dynamic system having multiple degrees of freedom, J. Appl. Mech. 30E, 367-372 (1963)

[11] N. W. McLachlan, Ordinary non-linear differential equations in engineering and physical sciences (2nd ed.), Clarendon Press, 1956

[12] W. Magnus and S. Winkler, Hill's equation, Interscience Publishers, 1966

[13] J. K. Moser, On invariant curves of area-preserving mappings of an annulus, Nachr. Akad. Wiss. Göttingen Math.-Phys. Kl. II, Nr. 1 (1962)

[14] C. H. Pak and R. M. Rosenberg, On the existence of normal mode vibrations in nonlinear systems, Quart. Appl. Math. 26, 403-416 (1968)

[15] G. Pecelli and E. Thomas, An example of elliptic stability with large parameters: Lamé's equation and the Arnold-Moser-Rüssmann criterion, Quart. Appl. Math. 36, 129-140 (1978)

[16] G. Pecelli and E. S. Thomas, On a class of modes defined by Rosenberg, to appear

[17] R. M. Rosenberg, On the existence of normal mode vibrations of nonlinear systems with two degrees of freedom, Quart. Appl. Math. 22, 217-234 (1964)

[18] R. M. Rosenberg and C. P. Atkinson, On the natural modes and their stability of non-linear two-degreeof-freedom systems, J. Appl. Mech. 26, 377-385, (1959)

[19] R. M. Rosenberg and J. K. Kuo, Nonsimilar normal mode vibrations of nonlinear systems having two degrees of freedom, J. Appl. Mech. 31E, 283-290 (1964)

[20] H. Rüssmann, Über die Normalform analytischer Hamiltonscher Differentialgleichungen in der Nähe einer Gleichgewichtslösung, Math. Annalen 19, 55-72 (1967)

[21] H. D. Seifert, Periodische Bewegungen mechanischer Systeme, Math. Zeit. 51, 197-216 (1947)

[22] C. L. Siegel and J. K. Moser, Lectures on celestial mechanics, Springer, Grundlehren Bd. 187 (1971)

[23] A. Weinstein, Normal modes for nonlinear Hamiltonian systems, Inv. Math. 20, 47-57 (1973) 